\title{
Drosophila Embryos as a Model for Wound-Induced Transcriptional Dynamics: Genetic Strategies to Achieve a Localized Wound Response
}

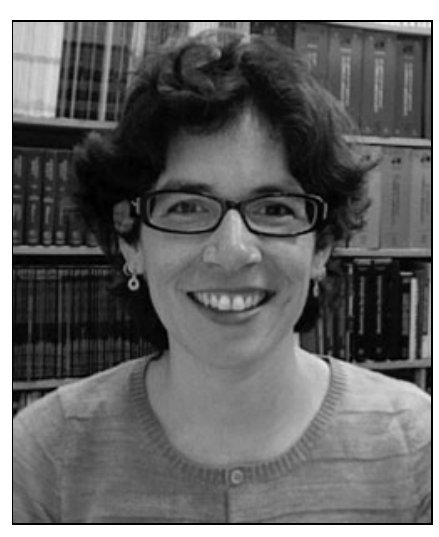

Michelle T. Juarez, PhD

Submitted for publication April 1, 2014. Accepted in revised form May 21, 2014.

${ }^{*}$ Correspondence: Sophie Davis School of Biomedical Education, City College New York, 160 Convent Ave., MR-510, New York, NY 10031 (e-mail: mjuarez@med.cuny.edu).

\author{
Michelle T. Juarez* \\ Sophie Davis School of Biomedical Education, City College of New York, New York, New York
}

While many studies have established a paradigm for tissue repair at the level of cellular remodeling, it is not clear how an organism restricts a response only to the injured region of a damaged tissue. Skin, the largest organ in the human body, is prone to injury, and repair of epidermal tissue represents a medically relevant system to investigate.

Significance: Studies in Drosophila melanogaster provide a robust genetic system to identify molecular components that will positively impact repair and healing. The Drosophila skin consists of a single-cell epidermal layer and relies on well-conserved cellular mechanisms to coordinate gene expression during development. Many studies have established that key developmental genes promote a response to epidermal injury, but the balance between activator and inhibitor signals to coordinate a localized response remains unknown.

Recent Advances: Discovery of a genetic pathway that promotes the restriction of transcriptional response to damage only in effected regions. Interestingly, genome-wide microarray studies have identified an intersection between gene expression after aseptic injury and activation of the innate immune response.

Critical Issues: The use of a transcriptional activation reporter provides an innovative approach to uncover well-conserved components that promote the localization of a response during epidermal injury and may influence other pathological conditions of tissue damage.

Future Directions: The work reviewed in this critical review may lead to development of molecular strategies of repair and improved healing after injury or infection. The outcomes on the fundamental contribution of a transcriptional response to injury will be translatable to mammalian systems.

\section{SCOPE AND SIGNIFICANCE}

DROSOPHILA PROVIDES a rich system to quickly assess complex biological processes. A combination of classical genetic techniques with advances in genome analyses and high-resolution microscopy enable discrete hypotheses to be tested on a diverse sample of molecular and cellular functions. The scope of this critical review is to highlight the contribution of a diverse collection of genes during a wound response to epidermal puncture injury in the Drosophila embryo. The significance of these studies is the identification of a novel set of wellconserved genes regulating the localization of a transcriptional response to injury.

\section{TRANSLATIONAL RELEVANCE}

In recent years, reports of patients suffering from chronic wound conditions have surpassed 6 million cases and this does not include the millions more recovering from surgical or traumatic wounds. ${ }^{1}$ Dissection of evolutionarily conserved genetic functions 
in model organisms is a key strategy in biomedical science to understand the basic cellular and molecular mechanisms. Studies using Drosophila as a model organism have contributed to the discovery of well-conserved regulatory pathways-including Hox-transcription factors during developmental patterning and Toll-signaling during innate immunity. Drosophila provides a robust system to elucidate conserved pathways that contribute to repair and regeneration.

\section{CLINICAL RELEVANCE}

The established paradigm for distinct phases of wound repair that promote healing and regeneration highlights the contributions of wound response, inflammation, and reepithelialization. ${ }^{2}$ Epidermal wound healing represents a powerful system to address a wide range of medically relevant pathologies. ${ }^{3}$ The regulation of activating and inhibiting signals is required for wound repair, however, the mechanisms of limiting the signals is poorly understood. ${ }^{4}$ This critical review provides an update on the functions of novel set of genes that regulate the localization of wound signals and may provide new directions to take clinical studies of wound care.

\section{BACKGROUND}

The single-layered epidermis in Drosophila provides a simple system to study an epidermal wound response after a puncture injury. ${ }^{5}$ Drosophila embryogenesis is a robust developmental stage to genetically dissect the phases of wound repair, including wound response, inflammation, and reepithelialization. ${ }^{6}$ This is in part because many or most zygotic mutants survive to the end of embryogenesis and develop epidermal barriers even when developmental patterning is defective. The development of the Drosophila epidermal barrier depends on the cross-linking of proteins, lipids, and chitin. ${ }^{7}$ Reactive quinones, the end product of a dopamine biosynthesis pathway, are also required for the maturation of the Drosophila epidermal barrier. ${ }^{8}$ Previous characterization of a well-conserved transcription factor grainy head (Grh), identified that Grh-target genes Dopa decarboxylase $(D d c)$ and tyrosine hydroxylase ( ple) are not only required during Drosophila epidermal barrier formation, but also are transcriptionally activated around the sites of injury. ${ }^{9}$ Drosophila as a model organism for wound response provides a complementary line of investigation to advance studies in mammalian wound healing. Mouse Grhlike3 gene is required epidermal barrier development and keratinocyte migration after injury. ${ }^{10} \mathrm{In}$
Drosophila there is one copy of GRH and in mouse there are three copies of GRH. Drosophila GRH is an essential protein, grh mutant embryos have weakened epidermal barrier development and do not survive outside of the protective vitelline membrane. ${ }^{9}$ In the Drosophila genome, the $5^{\prime}$ untranslated region (UTR) of several genes contain a conserved enhancer region with GRH transcription factor binding sites. A fluorescent gene coding sequence was cloned immediately downstream of the enhancer $5^{\prime}$ UTR and the resulting transgene generated an in vivo epidermal wound response reporter $^{11}$ (Fig. 1). These studies have developed a "toolkit" of many fluorescent wound reporters to monitor the in vivo response to epidermal injury in Drosophila embryos. ${ }^{12}$ Table 1 summarizes the epidermal wound reporters in two components of the dopamine biosynthesis pathway ( $D d c$ and ple), and two other genes required for barrier function: (1) misshapen (msn) encodes a Drosophila JNK kinase-kinase-kinase, ${ }^{13}$ (2) krotzkopf verkehrt (kkv) encodes a chitin synthase. ${ }^{14}$ The fluorescent reporters are active during the final stages of Drosophila embryo development. The early timing of the wound response reporter activity allows for localization patterns to be determined in mutants for essential genes that will not survive past embryogenesis (e.g., grh).

\section{Epidermal wound response gene detection}

Visualization of the wound response gene activation can be achieved by (1) in situ hybridization of fluorescently labeled RNA probes or (2) in vivo with enhancers controlling the expression of fluorescent reporter genes. For example, $D d c .47$ is a $470 \mathrm{bp}$ enhancer of Dopa decarboxylase cloned upstream of GFP and ple WE1 is a $3.0 \mathrm{~kb}$ enhancer of tyrosine hydroxylase cloned upstream of DsRed. Compound fluorescence microscopy can be used to quickly screen data and confocal fluorescence microscopy can be used for more detailed analysis. All of the wounding experiments are performed during the final stages of Drosophila development (stage 16 out of 17 total). ${ }^{15}$ The developing cuticle, in late stage Drosophila embryos, consists of stratified layers of chitin and makes detection of RNA transcripts a difficult task. A basic protocol for fluorescent in situ hybridization ${ }^{16}$ is adapted for enhancing the RNA detection. The RNA transcripts are visualized using a peroxidase enzyme amplification of the fluorescence signal.

The RNA transcripts of epidermal wound response genes can be detected within 30 min after a puncture wound. The wound reporter signal is slightly delayed because it requires the translation 


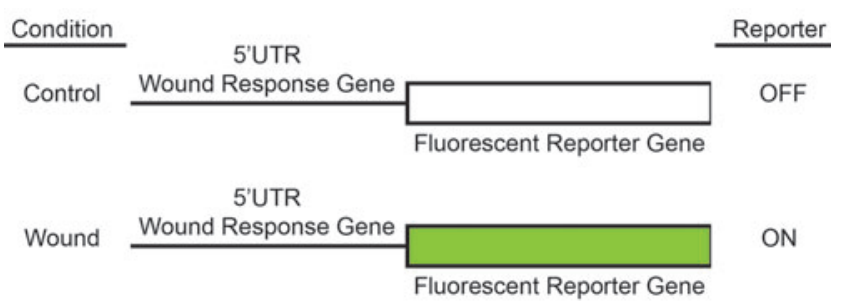

Figure 1. Architecture of epidermal wound response reporters. Activity of the reporter correlates with the condition of the embryo: control=OFF and wound $=0 \mathrm{~N}$. The $5^{\prime} U T R$ contains an enhancer region that is sufficient to activate fluorescent gene reporter expression and is dependent on puncture injury. Ddc, Dopa decarboxylase; UTR. To see this illustration in color, the reader is referred to the web version of this article at www.liebertpub.com/wound of the transgenic GFP and DsRed fluorescence genes. ${ }^{17}$ The localization of the $D d c$ and ple wound response reporters can be detected $4-6 \mathrm{~h}$ after puncture wounding. The epidermal wound reporter fluorescence is in the same pattern as the RNA transcripts of the wound response genes. The wound reporters provide an in vivo detection method for the transcriptional activation of the epidermal wound response genes. Using an in vivo detection is optimal for initiating genetic studies and the in situ detection is optimal for testing specific time points for gene expression.
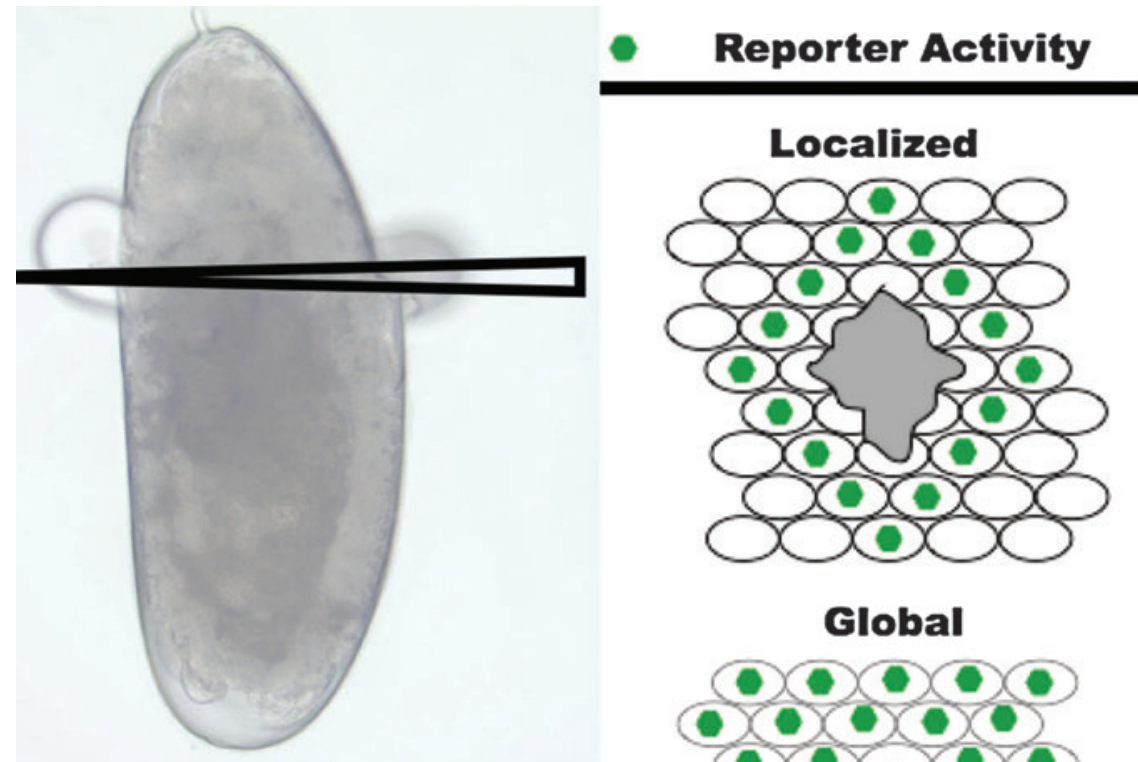

\section{Global}

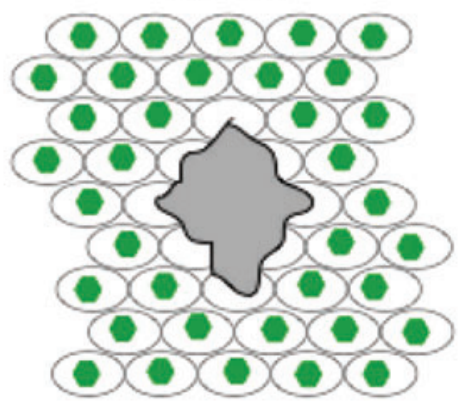

Absent

*

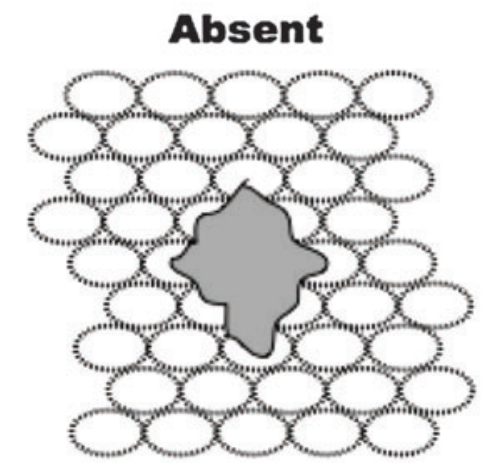

Figure 2. Microinjection injury and phenotypes of epidermal wound response reporters. Brightfield image with a line superimposed over the embryo to show the microinjection injury. Fluorescent image of the Ddc-GFP wound response reporter activity in the cells surrounding the site of puncture injury. A representation of the epidermal cells with an injury site; summarizing "localized," "global," or "absent" pattern of reporter activity. Asterisk marks the site of puncture injury. To see this illustration in color, the reader is referred to the web version of this article at www.liebertpub.com/wound 
Table 1. Drosophila epidermal wound response reporters

\begin{tabular}{lc}
\hline Epidermal Wound Response Reporters & Fluorescence Protein \\
\hline Dopa decarboxylase (Ddc), cuticle protein & GFP \\
Tyrosine hydroxylase (ple), cuticle protein & DsRed \\
Misshapen (msn), JNK-KKK & DsRed \\
Krotzkopf verkehrt (kkv), chitin synthase & DsRed \\
\hline
\end{tabular}

\section{Genetic regulation of an epidermal wound response}

Recent reports on the regulation of Drosophila wound healing have focused on the wound closure phenotype, allowing discovery of many pathways regulating cell migration. ${ }^{18,19}$ The fluorescent wound reporter localization pattern provides a direct assay of transcriptional activation of the barrier repair genes after microinjection injury (Fig. 2). The fluorescent wound reporter localization was used in an unbiased screen of $\sim 5,000$ mutant genes to identify a novel set of well-conserved genes required for the localized expression of epidermal wound-inducible genes $^{20}$ (Table 2). Subsequent studies to complement the genetic screen, used a microarray to survey the transcriptional profiles of gene expression after puncture injury. ${ }^{21}$ In previous microarray studies using a laser-mediated wounding technique in Drosophila embryos, the enrichment levels of gene expression for control compared to wounded samples was not significantly different. ${ }^{22}$ To address the issue of a puncture wound resulting in a small area of damaged cells compared to the whole embryo, a novel protocol was developed for a simultaneous puncture and chemical microinjection of a serine protease, Trypsin. Serine proteases play an important role in the signal transduction pathways controlling dorsovental patterning, innate immune response, and localization of the epidermal wound response. ${ }^{20,23,24}$ The combination of puncture injury and trypsinmediated activation resulted in the identification of a novel set of eight genes that are transcriptionally upregulated in the epidermal cells surrounding a puncture injury in the Drosophila embryo (Ady43A, Ets at $21 \mathrm{C}$, jun-related antigen [jra/jun], kayak [fos], Relish, rhomboid, spatzle, and dorsal). ${ }^{21}$

Table 2. Genes that regulate the localization of epidermal wound response reporters

\begin{tabular}{lc}
\hline New Genes Required for Localized Wound Reporter & Phenotype \\
\hline Flotillin-2, lipid raft-associated protein (cg32593) & Global \\
Src42A, protein tyrosine kinase (cg7873) & Global \\
Dual oxidase, NADPH oxidase and peroxidase (cg3131) & Absent \\
Wurst, Clathrin-associated protein (cg9098) & Global \\
Ghost, COPII vesicle component (cg10882) & Absent \\
Varicose, septate junction- associated protein (cg 9326) & Global \\
Drosophila homolog of yeast Maki (cg11412) & Global \\
Shroud, short-chain dehydrogenase/reductase (cg12068) & Absent \\
\hline
\end{tabular}

Comparing the conservation of genes upregulated in recent microarray studies in mammalian wound repair with the results from the Drosophila microarray presented in this critical review studies highlight key similarities and differences between the distinct model systems. A prominent example of conserved gene regulatory profiles was observed in the significant upregulation of the FOS and JUN family genes. ${ }^{21,25-27}$ In addition, downstream effectors of the JNK signaling pathway have welldescribed mutant phenotypes that disrupt wound healing in the Drosophila embryo. ${ }^{28,29}$ During the reepithelialization stage of wound repair, mammalian cells undergo a process of cellular proliferation. ${ }^{30}$ In contrast, Drosophila embryonic epidermal cells resolve a wound through migration of neighboring cells. ${ }^{31} \mathrm{~A}$ distinct mode of transcriptional regulation between mammalian and Drosophila wound repair can be observed in the regulation of genes promoting cell cycle. For example, several cyclin genes were significantly downregulated after puncture in Drosophila embryos. ${ }^{21}$ A similar class of cyclin genes were significantly upregulated after mammalian keratinocyte scratch wounding. ${ }^{26}$ It is interesting that the recent microarray studies in the Drosophila embryo identified Src $42 A$ as being upregulated after puncture injury, however, Flo-2 was not identified. ${ }^{21}$ This result suggests a sensitivity limitation of the microarray experiment and the challenge of detecting transcriptional changes in gene like Flo-2, which is highly expressed in all tissues in the developing embryo. ${ }^{20}$

\section{DISCUSSION OF FINDINGS AND RELEVANT LITERATURE}

From the list of novel genes identified in the initial genetic screen, three genes (Flotillin-2, Src42A, and Dual Oxidase) were of significant interest because of their previously published roles in regeneration, signal transduction, and wound response signaling. Flotillins were originally identified as a novel class of membrane-associated proteins in detergent resistant fractions purified from mammalian cells. ${ }^{32}$ Another group characterized flotillins as being upregulated in regenerating optic neurons of injured goldfish retinal ganglion cells. ${ }^{33}$ Flotillins are highly conserved among metazoan animals, 80\% similarity among vertebrates and invertebrates. ${ }^{34}$ Previous work to characterize Drosophila Flotillins suggest a molecular mechanism in cell-cell communication. ${ }^{35,36}$ The "global" pattern of the wound reporter in the loss-of-function Flo-2 mutant embryo and the "absent" pattern of the wound reporter in the gain-of- 
function Flo-2 mutant embryo suggest that Flo-2 is both necessary and sufficient to limit the spread of the epidermal wound response reporter activity. A similar "global" pattern of RNA localization were observed in Flo-2 mutants with in situ results of $D d c$, ple, msn, and $k k v .^{20}$ The Flo-2 mutant embryos do not have a wound healing phenotype and survival rates after wounding for both the loss-offunction and gain-of-function Flo-2 mutants show similar rates to wounded wild-type controls. Of particular interest to the study of signal transduction during the wound response is the previous report that flotillins require phosphorylation mediated by Src-family kinases to maintain localization at the plasma membrane of cultured mammalian neuronal cells. ${ }^{37}$ Src-family kinases are evolutionarily conserved group of protein tyrosine kinases that promote cellular proliferation and were recently shown to integrate wound response signals during zebrafish tissue regeneration. ${ }^{38}$. Consistent with the previous reports that flotillins depend on the function Src-family kinase activity, similar "global" pattern of epidermal wound reporter localization are observed in Src42A loss-of-function mutant embryos and "absent" pattern of the wound reporter in the gain-of-function Src42A mutant embryo. However, loss-of-function $S r c 42 A$ mutants to do complete embryogenesis and survival rates after wounding cannot be determined. Future studies that focus on the cellular localization of Flo-2 epidermal cells surrounding the site of injury may define a role for Src phosphorylation and signal transduction during the transcriptional activation of wound response genes. A balance in signal localization may improve how an organism coordinates an "activator" response for repair and an "inhibitor" response for scarring or inflammation. The Drosophila embryo provides a developmental system to correlate the studies of wound reporter localization in combination with cellular localization. One outcome of these studies is that Flo-2 can contribute to a better understanding of wound signal dynamics and lead to improving tissue repair treatments.

During injury, damaged cells produce multiple signals to elicit a wound response. Additional work using a model of zebrafish regeneration developed the use of a hydrogen peroxide sensor correlated with the migration of inflammatory cells to the site of injury. ${ }^{39}$ Similar results were observed in Drosophila and genetic tests indicated the enzyme dual oxidase (Duox) is required for the hydrogen peroxide production and stimulation of the inflammatory response. ${ }^{40}$ Recent studies from Drosophila showed that Duox can also promote a calcium flux at the site of epidermal injury. ${ }^{41}$ Additional genetic analyses combine the localization of the epidermal wound response reporters with the activity of chemical compounds (e.g., Serine protease-trypsin, hydrogen peroxide, and Src-Kinase inhibitorSU6656). Interestingly, the overexpression of Flo-2 and $\operatorname{Src} 42 A$ are sufficient to inhibit not only the activation of a local puncture wound response, but also the global activation of a chemical-mediated wound response (e.g., trypsin and hydrogen peroxide). ${ }^{20} \mathrm{~A}$ model is proposed for novel interactions between Flo-2, Src42A, and Duox and (Fig. 3).

New insight about the mechanism for localization of the wound response reporters in the cells surrounding injury can be inferred from the other novel genes identified in the genetic screen (Table 2) and with support from online resources (flybase.org). ${ }^{42,43}$

Several of the genes that have mutant phenotypes in wound response localization also have phenotypes in tracheal tube formation. Similarities between the localization of wound reporters and tracheal tube development exist between processes that communicate signals to coordinate

Dual Oxidase (Hydrogen peroxide)

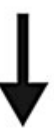

Serine protease (Trypsin)

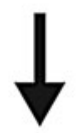

Grainy head

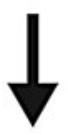

Epidermal Wound Gene Activation

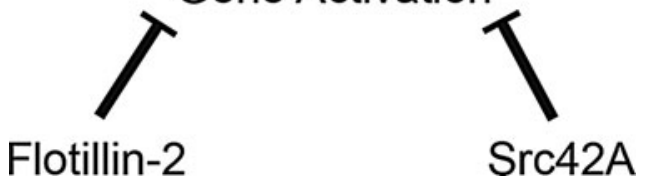

Figure 3. Key components of genetic pathway regulating epidermal wound response reporters. Epistatic relationships were determined using genetic analyses of double mutants and chemical-mediated wound response reporter activation. The pathway is not linear and receives overlapping signals from multiple components. 
expansion of wound response or tube size. In addition, several proteins that promote clearance removal of cellular materials in the interior of the tracheal tube may determine the turnover of the wound response. Wurst is a transmembrane protein that promotes liquid-clearance and air-filling during tracheal tube development and achieves this essential conversion through clathrin-associated endocytosis. ${ }^{44}$ Wurst mutant embryos have a "global" pattern of wound reporter localization and suggest that defects in endocytosis and clearance of a wound signal may contribute to the phenotype. Additional support for endocytosis as a cellular process to limit the spread of a wound signal come from research on the role of flotillins in cell signaling during regulatory processes involved in regeneration. ${ }^{45}$ Both Flo-2 and wurst mutant embryos have the "global" pattern of wound localization after injury. Recent work on flotillin function in both plants and bacteria highlight a role in endocytosis. ${ }^{46,47}$ Rab proteins are key components of the endocytic pathway and have a well-established role in coordinating development ${ }^{48}$ and may provide an intersection between wound response localization and endocytosis. Future studies to determine the contribution of endocytic pathway components and their distinct localization patterns during epidermal wound response in the Drosophila embryo may provide further insight into cellular mechanism promoting wound repair.
An important function of a critical review is promoting scientific transparency and maintaining a constant review of the research literature. New genomic data can resolve closely mapped genes that were previously thought to be alleles of the same gene (e.g., kayak and shroud). Based on a previous study, the kayak-shroud allele was in the Drosophila FosD gene. ${ }^{49}$ Initial studies observed an epidermal wound response reporter phenotype in mutant embryos of the kayak-shroud allele. ${ }^{11}$ Subsequent studies determined that the shroud allele was a mutation in a neighboring gene, encoding a short-chain dehydrogenase/reductase. ${ }^{50} \mathrm{It}$ is clear that shroud plays an important role in regulating wound repair from the original study of the wound reporter localization defects. ${ }^{11}$ The broader role of hormone biosynthesis in the context of wound repair remains to be determined. Recent studies have found new evidence to support the claim that kayak plays an important role in regulating not only the localization of the epidermal wound response, ${ }^{21}$ but also promotes epidermal cell migration during wound healing. ${ }^{19,51}$

During the procedure of microinjection with a glass pipette, no additional techniques are employed to preserve a sterile environment and the result is a localized pattern of wound reporter activation (Fig. 4). However, the direct puncture of a Drosophila embryo in a liquid culture of Gramnegative bacteria, Erwinia carotovora carotovora,
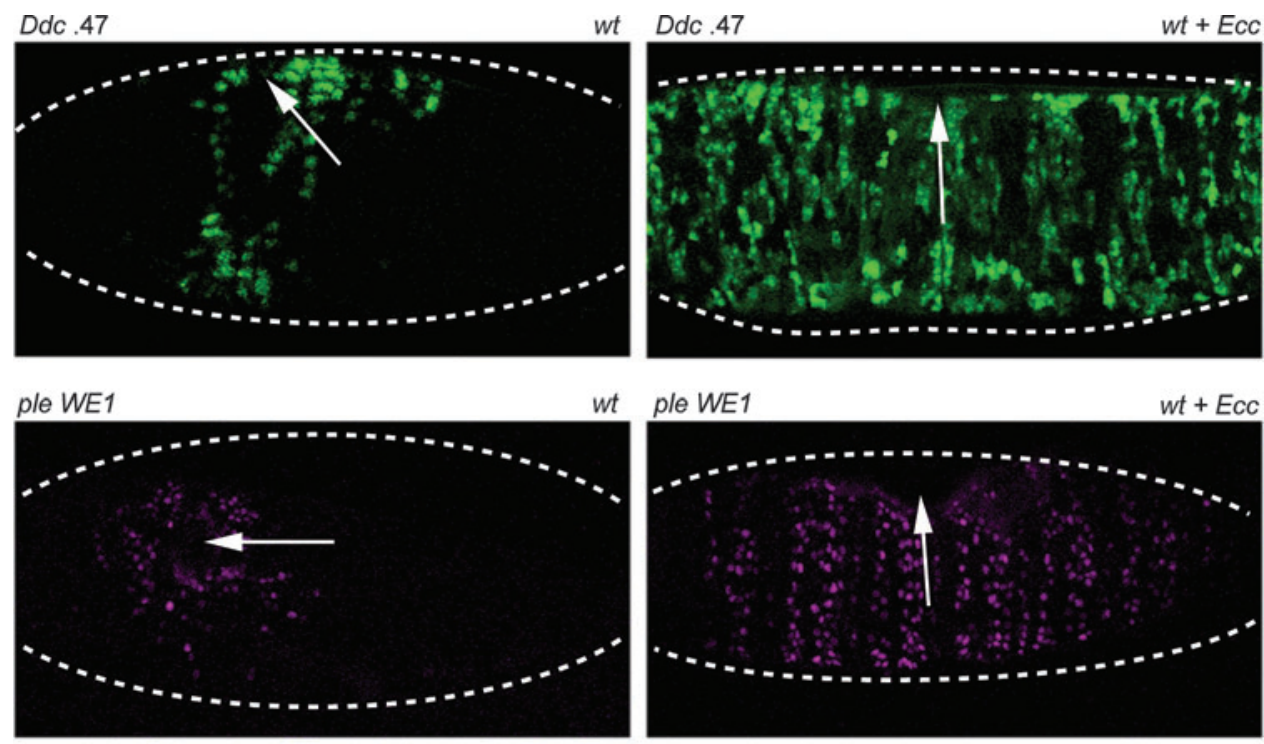

ple WE1

$w t+E c c$

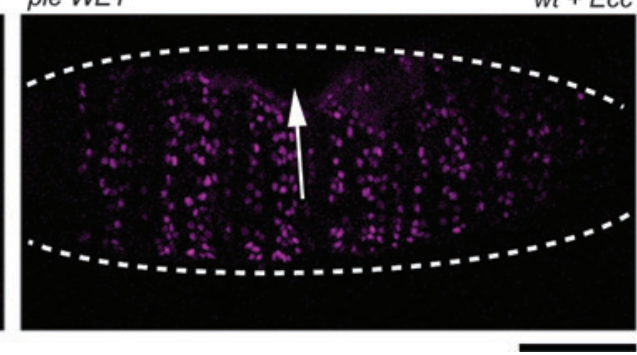

Figure 4. Aseptic and septic injury. Fluorescent images of embryos with Ddc.47 and ple WE1 wound response reporters. wild type (wt) is an aseptic condition and is the "standard" protocol for puncture injury. The aseptic condition activates a "localized" pattern of the wound response reporters. wt+Ecc is a septic condition and is achieved by injuring the embryo while submerged in a liquid culture of Erwina carotovora carotovora. The septic condition activates a "global" pattern of the wound response reporters. Dashed lines outline the embryo. Arrows mark the site of puncture injury. Scale bar $=50 \mu \mathrm{m}$. Ecc, Erwinia carotovora carotovora; ple, tyrosine hydroxylase. To see this illustration in color, the reader is referred to the web version of this article at www.liebertpub.com/wound 
can elicit a "global" pattern of wound reporter activation (Fig. 4). The comparison of punctureinjury and trypsin-mediated injury gene expression profiles provides additional evidence that aseptic injury regulates innate immune response. ${ }^{21} \mathrm{~A}$ recent article from the Medzhitov lab, an expert in the field of mammalian innate immunity, provides evidence that increased tissue repair can contribute to decreased mortality after secondary infection with bacterial pathogens in a mouse model of influenza infection in the upper respiratory tract. ${ }^{52}$ Future studies with Drosophila puncture injury may provide a direct model to test the hypothesis that a localization of damage signals will promote tissue repair.

\section{SUMMARY}

The Drosophila embryo has enlisted a wellconserved ensemble of genes to promote a local response to epidermal injury and suppress "global" response. Recent studies from cardiac injury studies in zebrafish provide additional support for the important role of balancing activating and inhibiting signals during heart regeneration. ${ }^{53} \mathrm{Ad}-$ ditional studies in planaria tissue regeneration demonstrate a wide range of inductive signals activate the neoblast cell population and contribute to a wound response pattern. ${ }^{54}$

The significance of the Drosophila epidermal wound response reporter method is to combine the protocol for puncture/microinjection with visualization of a transcriptional response to epidermal injury in Drosophila embryos. Survival after puncture wounding is greater than $95 \%$ in wild-type embryos. ${ }^{12}$ One of the merits of using transcriptional activation as a method to study wound repair is that the results provide more insight into a mechanism of how signals are transduced from the site of injury to the neighboring cells. Broader use of the aseptic wound puncture protocol in genetic and chemical screens will allow identification of new regulators of the transcriptional response to epidermal wounding, and further the translation of wound healing discoveries into mammalian models of injury treatments. However, one of the demerits of the transcriptional activation method is that the results do not directly link to phenotypes in wound healing or repair. For example, Drosophila Flotillin-2 mutant embryos heal after epidermal puncture injury. ${ }^{20}$

The studies highlighted in this critical review will provide insight on the epidermal wound response in Drosophila by answering questions pertaining to the cellular mechanism of wound signal localization and the intersection of wound repair and innate immunity. Results from these studies

\section{TAKE-HOME MESSAGES}

- A localized transcriptional response to injury is regulated by a set of well-conserved genes.

- Barrier formation genes promote development of protective layers and establishing a response to cellular damage

- Drosophila studies provide insight into novel functions of genes during the processes controlling a response to epidermal injury.

- Wound care may be improved by applying activators or inhibitors of a localized wound response pathway.

- The balance of activating a wound response and suppressing an immune response may improve wound repair

on Drosophila wound response can further impact other models of regeneration at both the level of animal systems (e.g., zebrafish) and human organ systems (e.g., skin). Recent studies in zebrafish show a similar role for oxidative species (e.g., hydrogen peroxide) in promoting wound repair and regeneration that complement the preliminary studies with a role for the enzyme Duox in wound response activation. ${ }^{20,39}$ In addition, the original report on the role of flotillins in optic nerve regeneration in goldfish has been confirmed in zebrafish and morpholino experiments show a specific requirement for flotillin activity during this process. ${ }^{55}$ For the application of the Drosophila wound response results to human models of regeneration and repair, many models of both developmental pathologies as well as induced injury cases exist. The recent availability of a Flo- 1 knockout mouse model will allow bridging experiments to be performed and test the efficacy of the result from Drosophila studies. ${ }^{56}$ New insights into the complex regulation of the transcriptional response after injury in the Drosophila embryo will improve paradigms for tissue repair and regeneration in clinically relevant models of cellular damage.

\section{ACKNOWLEDGMENTS AND FUNDING SOURCES}

M.T. Juarez currently receives support from a CCNY-Research Centers in Minority Institutions (RCMI) Grant. The project described was supported by Grant Number 5G12RR003060-26 from the National Center for Research Resources and Grant Number 8G12MD7603-28 from the National Institute on Minority Health and Health Disparities. The content is solely the responsibility of the authors and does not necessarily represent the official views of the National Institute on Minority Health and Health Disparities or the National Institutes of Health. 


\section{AUTHOR DISCLOSURE AND GHOSTWRITING}

No competing financial interests exist. The content of this article was expressly written by the author listed. No ghostwriters were used to write this article.

\section{ABOUT THE AUTHOR}

Michelle T. Juarez, PhD, is an Assistant Medical Professor in the Pathobiology Depart- ment of the Sophie Davis School of Biomedical Education at the City College of New York. She received her B.S. from the University of California, Berkeley and her $\mathrm{PhD}$ from the State University of New York, Stony Brook. She completed her postdoctoral fellowship at the University of California, San Diego and worked with Dr. William McGinnis. Dr. Juarez has received support from the National Institutes of Health (R01 GM077197 and K12 GM68524).

\section{REFERENCES}

1. Sen CK, Gordillo GM, Roy S, Kirsner R, Lambert L, Hunt TK, et al. Human skin wounds: a major and snowballing threat to public health and the economy. Wound Repair Regen 2009;17:763-771.

2. Reinke JM, Sorg H. Wound repair and regeneration. Eur Surg Res 2012;49:35-43.

3. Demidova-Rice TN, Hamblin MR, Herman IM. Acute and impaired wound healing: pathophysiology and current methods for drug delivery, part 1: normal and chronic wounds: biology, causes, and approaches to care. Adv Skin Wound Care 2012;25:304-314.

4. Weber CE, Li NY, Wai PY, Kuo PC. Epithelialmesenchymal transition, TGF- $\beta$, and osteopontin in wound healing and tissue remodeling after injury. J Burn Care Res 2012;33:311-318.

5. Payre F. Genetic control of epidermis differentiation in Drosophila. Int J Dev Biol 2004;48:207-215.

6. Gurtner GC, Werner S, Barrandon Y, Longaker MT. Wound repair and regeneration. Nature 2008;453: 314-321.

7. Moussian B. Recent advances in understanding mechanisms of insect cuticle differentiation. Insect Biochem Mol Biol 2010;40:363-375.

8. Andersen S0. Insect cuticular sclerotization: a review. Insect Biochem Mol Biol 2010;40:166-178.

9. Mace KA, Pearson JC, McGinnis W. An epidermal barrier wound repair pathway in Drosophila is mediated by grainy head. Science 2005;308:381-385.

10. Ting SB, Caddy J, Hislop N, Wilanowski T, Auden A, Zhao L-L, et al. A homolog of Drosophila grainy head is essential for epidermal integrity in mice. Science 2005;308:411-413.

11. Pearson JC, Juarez MT, Kim M, Drivenes $\varnothing$, McGinnis W. Multiple transcription factor codes activate epidermal wound-response genes in Drosophila. Proc Natl Acad Sci U S A 2009;106: 2224-2229.

12. Juarez MT, Patterson RA, Li W, McGinnis W. Microinjection wound assay and in vivo localization of epidermal wound response reporters in Drosophila embryos. J Vis Exp 2013:e50750.

13. Weston CR, Davis RJ. The JNK signal transduction pathway. Curr Opin Cell Biol 2007;19:142-149.
14. Ostrowski S, Dierick HA, Bejsovec A. Genetic control of cuticle formation during embryonic development of Drosophila melanogaster. Genetics 2002;161:171-182.

15. Hartenstein V, Lee A, Toga AW. A graphic digital database of Drosophila embryogenesis. Trends Genet 1995;11:51-58.

16. Kosman D, Mizutani CM, Lemons D, Cox WG, McGinnis W, Bier E. Multiplex detection of RNA expression in Drosophila embryos. Science 2004; 305:846.

17. Barolo S, Castro B, Posakony JW. New Drosophila transgenic reporters: insulated P-element vectors expressing fast-maturing RFP. BioTechniques 2004;36:436-440, 442

18. Campos I, Geiger JA, Santos AC, Carlos V, Jacinto A. Genetic screen in Drosophila melanogaster uncovers a novel set of genes required for embryonic epithelial repair. Genetics 2010;184: 129-140.

19. Lesch C, Jo J, Wu Y, Fish GS, Galko MJ. A targeted UAS-RNAi screen in Drosophila larvae identifies wound closure genes regulating distinct cellular processes. Genetics 2010;186:943-957.

20. Juarez MT, Patterson RA, Sandoval-Guillen E, McGinnis W. Duox, Flotillin-2, and Src42A are required to activate or delimit the spread of the transcriptional response to epidermal wounds in Drosophila. PLoS Genet 2011;7:e1002424.

21. Patterson RA, Juarez MT, Hermann A, Sasik R, Hardiman G, McGinnis W. Serine proteolytic pathway activation reveals an expanded ensemble of wound response genes in Drosophila. PLoS One 2013;8:e61773.

22. Stramer B, Winfield $M$, Shaw $T$, Millard $T H$, Woolner S, Martin P. Gene induction following wounding of wild-type versus macrophagedeficient Drosophila embryos. EMBO Rep 2008;9: 465-471.

23. LeMosy EK, Hong CC, Hashimoto C. Signal transduction by a protease cascade. Trends Cell Biol 1999:9:102-107

24. Imler JL, Hoffmann JA. Signaling mechanisms in the antimicrobial host defense of Drosophila. Curr Opin Microbiol 2000;3:16-22.
25. Cooper L, Johnson C, Burslem F, Martin P. Wound healing and inflammation genes revealed by array analysis of 'macrophageless' PU.1 null mice. Genome Biol 2005;6:R5.

26. Fitsialos G, Chassot A-A, Turchi L, Dayem MA LeBrigand $\mathrm{K}$, Moreilhon $\mathrm{C}$, et al. Transcriptional signature of epidermal keratinocytes subjected to in vitro scratch wounding reveals selective roles for ERK1/2, p38, and phosphatidylinositol 3-kinase signaling pathways. J Biol Chem 2007;282: 15090-15102.

27. Cole J, Tsou R, Wallace K, Gibran N, Isik F. Early gene expression profile of human skin to injury using high-density cDNA microarrays. Wound Repair Regen 2001;9:360-370.

28. Galko MJ, Krasnow MA. Cellular and genetic analysis of wound healing in Drosophila larvae. PLoS Biol 2004;2:E239.

29. Rämet M, Lanot R, Zachary D, Manfruelli P. JNK signaling pathway is required for efficient wound healing in Drosophila. Dev Biol 2002;241:145-156.

30. Santoro MM, Gaudino G. Cellular and molecular facets of keratinocyte reepithelization during wound healing. Exp Cell Res 2005;304:274-286.

31. Wood W, Jacinto A, Grose R, Woolner S, Gale J, Wilson $C$, et al. Wound healing recapitulates morphogenesis in Drosophila embryos. Nat Cell Biol 2002;4:907-912.

32. Bickel PE, Scherer PE, Schnitzer JE, Oh P, Lisanti MP, Lodish HF. Flotillin and epidermal surface antigen define a new family of caveolae-associated integral membrane proteins. J Biol Chem 1997;272:13793-13802.

33. Schulte T, Paschke KA, Laessing U, Lottspeich F, Stuermer CA. Reggie-1 and reggie-2, two cell surface proteins expressed by retinal ganglion cells during axon regeneration. Dev Camb Engl 1997;124:577-587.

34. Galbiati F, Volonte D, Goltz JS, Steele Z, Sen J, Jurcsak J, et al. Identification, sequence and developmental expression of invertebrate flotillins from Drosophila melanogaster. Gene 1998;210: 229-237.

35. Hoehne M, de Couet HG, Stuermer CAO, Fischbach K-F. Loss- and gain-of-function analysis of 
the lipid raft proteins Reggie/Flotillin in Drosophila: they are posttranslationally regulated, and misexpression interferes with wing and eye development. Mol Cell Neurosci 2005;30:326-338.

36. Katanaev VL, Solis GP, Hausmann G, Buestorf S, Katanayeva N, Schrock Y, et al. Reggie-1/flotillin2 promotes secretion of the long-range signalling forms of Wingless and Hedgehog in Drosophila. EMBO J 2008:27:509-521.

37. Neumann-Giesen C, Fernow I, Amaddii M, Tikkanen R. Role of EGF-induced tyrosine phosphorylation of reggie-1/flotillin-2 in cell spreading and signaling to the actin cytoskeleton. J Cell Sci 2007;120:395-406.

38. Yoo SK, Freisinger CM, LeBert DC, Huttenlocher A. Early redox, Src family kinase, and calcium signaling integrate wound responses and tissue regeneration in zebrafish. J Cell Biol 2012;199: 225-234.

39. Niethammer P, Grabher C, Look AT, Mitchison TJ. A tissue-scale gradient of hydrogen peroxide mediates rapid wound detection in zebrafish. Nature 2009;459:996-999.

40. Moreira S, Stramer B, Evans I, Wood W, Martin P. Prioritization of competing damage and developmental signals by migrating macrophages in the Drosophila embryo. Curr Biol 2010;20:464-470.

41. Razzell W, Evans IR, Martin P, Wood W. Calcium flashes orchestrate the wound inflammatory response through DUOX activation and hydrogen peroxide release. Curr Biol 2013;23:424-429.

42. McQuilton P, St. Pierre SE, Thurmond J. FlyBase 101-the basics of navigating FlyBase. Nucleic Acids Res 2012;40:D706-D714.
43. St Pierre SE, Ponting L, Stefancsik R, McQuilton P, FlyBase Consortium. FlyBase 102-advanced approaches to interrogating FlyBase. Nucleic Acids Res 2014;42:D780-D788.

44. Stümpges B, Behr M. Time-specific regulation of airway clearance by the Drosophila J-domain transmembrane protein Wurst. FEBS Lett 2011; 585:3316-3321.

45. Stuermer CAO. How reggies regulate regeneration and axon growth. Cell Tissue Res 2012;349: 71-77.

46. Dempwolff F, Wischhusen HM, Specht M, Graumann PL. The deletion of bacterial dynamin and flotillin genes results in pleiotrophic effects on cell division, cell growth and in cell shape maintenance. BMC Microbiol 2012;12:298.

47. Haney CH, Long SR. Plant flotillins are required for infection by nitrogen-fixing bacteria. Proc Nat Acad Sci U S A 2010:107:478-483.

48. Ricard CS, Jakubowski JM, Verbsky JW, Barbieri MA, Lewis WM, Fernandez GE, et al. Drosophila rab GDI mutants disrupt development but have normal Rab membrane extraction. Genesis 2001; 31:17-29.

49. Giesen K, Lammel U, Langehans D, Krukkert K, Bunse I, Klämbt C. Regulation of glial cell number and differentiation by ecdysone and Fos signaling. Mech Dev 2003:120:401-413.

50. Niwa R, Namiki T, Ito K, Shimada-Niwa Y, Kiuchi M, Kawaoka S, et al. Non-molting glossy/shroud encodes a short-chain dehydrogenase/reductase that functions in the 'Black Box' of the ecdysteroid biosynthesis pathway. Dev Camb Engl 2010;137: 1991-1999.
51. Brock AR, Wang Y, Berger S, Renkawitz-Pohl R, Han VC, Wu Y, et al. Transcriptional regulation of Profilin during wound closure in Drosophila larvae. J Cell Sci 2012;125:5667-5676.

52. Jamieson AM, Pasman L, Yu S, Gamradt P, Homer RJ, Decker $T$, et al. Role of tissue protection in lethal respiratory viral-bacterial coinfection. Science 2013;340:1230-1234.

53. Gupta V, Gemberling M, Karra R, Rosenfeld GE, Evans T, Poss KD. An injury-responsive gata4 program shapes the zebrafish cardiac ventricle. Curr Biol 2013;23:1221-1227.

54. Wenemoser D, Lapan SW, Wilkinson AW, Bell GW, Reddien PW. A molecular wound response program associated with regeneration initiation in planarians. Genes Dev 2012;26:988-1002.

55. Munderloh C, Solis GP, Bodrikov V, Jaeger FA, Wiechers M, Málaga-Trillo E, et al. Reggies/flotillins regulate retinal axon regeneration in the zebrafish optic nerve and differentiation of hippocampal and N2a neurons. J Neurosci 2009;29:6607-6615.

56. Ludwig A, Otto GP, Riento K, Hams E, Fallon PG, Nichols BJ. Flotillin microdomains interact with the cortical cytoskeleton to control uropod formation and neutrophil recruitment. J Cell Biol 2010;191:771-781.

\section{Abbreviations and Acronyms}

Ddc $=$ Dopa decarboxylase

Duox $=$ dual oxidase

Flo-2 = flotillin-2

$\mathrm{Grh}=$ grainy head

ple $=$ tyrosine hydroxylase

UTR $=$ untranslated region 